\title{
A Case of Ciprofloxacin-induced Acute Psychosis After Aortic Dissection Surgery
}

\section{Guobao Song}

Second Xiangya Hospital

\section{Bo Jiang}

Second Xiangya Hospital

Shenglan Tan

Second Xiangya Hospital

Feng Li ( $\nabla$ muzicn@hotmail.com )

Second Xiangya Hospital

\section{Case report}

Keywords: Ciprofloxacin, Psychosis, FDA hypothesis

Posted Date: September 2nd, 2020

DOl: https://doi.org/10.21203/rs.3.rs-68873/v1

License: (c) (1) This work is licensed under a Creative Commons Attribution 4.0 International License. Read Full License 


\section{Abstract}

Ciprofloxacin is widely used in general infected population, including patients with aortic dissection. Ciprofloxacin-mediated neurotoxicity has been observed in few reports, and some studies found ciprofloxacin may increase the incidence of aortic dissection or aortic aneurysm rupture. We report here a case of ciprofloxacin-induced acute psychosis after Sun's total arch replacement and cardiopulmonary resuscitation. Based on the published literature and FDA hypothesis, we suggest that ciprofloxacin should be used cautiously in patients after aortic dissection surgery in the risk of drug-induced psychosis and residual dilated aortic rupture.

\section{Case Report}

A 39-year-old male presented with sudden chest and back tearing-like pain for 11 hours. The past history included more than 10 years of chronic hepatitis B, and the history of cerebrovascular disease and mental illness was denied. We confirmed his diagnosis as acute type $A$ aortic dissection (AAD). The patient underwent aortic root reconstruction, ascending aorta replacement, total arch replacement and stented elephant trunk implantation (Sun's procedure). During the hemostasis, the patient had cardiac arrest. After cardiopulmonary resuscitation (CPR), the patient recovered stable hemodynamics and was given sub-hibernation therapy on the same day after the operation. He was conscious on day 3 and stopped sub-hibernation therapy on day 5. Postoperative echocardiography demonstrated a normal ejection fraction of $73 \%$. CT angiography (CTA) of aorta showed normal head and neck vessels and bilateral lung exudation.

The patient then developed a fever and severe pulmonary infection. Pseudomonas aeruginosa and Klebsiella pneumoniae were found in sputum culture from 6 to 22 days. The highest white blood cell count (WBC) exceeding $30 * 10^{9} / \mathrm{L}$ on day 13 . Under the guidance of clinical pharmacists, we used imipenem combined with vancomycin, meropenem combined with linezolid and caspofungin successively. The patient's condition gradually improved and his fever subsided. The white blood cell count decreased to normal on day 19 . According to the drug sensitivity test, piperacillin and ciprofloxacin (dosage: $200 \mathrm{mg} / \mathrm{time}$, bid, intravenous drip) were used on day 23. The patient had a clear mind and a stable breath that day. Two days later, the patient appeared to be talkative, restless, and agitated. Obvious delirium was found: he kept wriggling in bed all the time; questions could be answered, but sometimes answers were quite irrelevant; talking gibberish; suspected persecutory delusions because he always said "call 110 to the police"; denied hallucinations, and stayed up all night. No abnormality was found in emergent head CT and total aorta CTA. WBC count, procalcitonin, blood gas analysis, electrolytes, liver function and kidney function were basically normal. The psychiatrist considered suspected drug-induced delirium after consultation. He suggested that pharmacists should be consulted to adjust the antibiotics. A pharmacist considered a possible diagnosis of acute ciprofloxacin-induced psychosis. We replaced ciprofloxacin with amikacin on day 26 . We also gave him rehydration therapy, vitamin B6, and quetiapine (25 mg per night) for five days. After stopping ciprofloxacin for a week, he returned to normal cognitive state. 


\section{Discussion}

Ciprofloxacin is the third generation of fluoroquinolones. It is among the most commonly prescribed antibiotics and is well-tolerated in adults. The general adverse effects are gastrointestinal reactions. Ciprofloxacin also has some rare side effects observed, including palpitation and prolonged QT interval in cardiovascular system, acute granulocytopenia in blood system, tendonitis and tendon ruptures. In addition, the adverse events in the central nervous system (CNS) have been sporadically reported including mental disorders, transient motor aphasia, epilepsy, and confusion etc. Grimm et al. ${ }^{[1]}$ described a case of ciprofloxacin-induced acute polymorphic psychosis with strong persecutory delusions. They gave the patient lorazepame for the first week, then began antipsychotic treatment with aripiprazole for 9 days, until the patient was free from the ideas of persecution. Ben-Chetrit et al. ${ }^{[2]}$ reported an old male accepted ciprofloxacin for 4 days then experienced visual delusions and loss of orientation in time and place. Another report ${ }^{[3]}$ presented a woman, received ciprofloxacin for 6 days, developed fearfulness, psychomotor retardation, visual and auditory hallucinations. The symptoms of above two patients resolved after ciprofloxacin withdrawal, without any additional intervention.

The mechanism of ciprofloxacin induced psychosis remains unclear. In an animal research, Kaur et al. [4] found that compared with controls, all ciprofloxacin treatment groups showed higher levels of apoptosis in cerebral cortex. In other in vitro studies, ${ }^{[5]}$ protein linked double-stranded DNA was broken in ciprofloxacin treated cells, indicating that ciprofloxacin targets mitochondrial topoisomerase II activity. This inhibition effect results in decreased mitochondrial respiration, followed by extensive oxidative damage in the brain and further neuropsychiatric symptoms.

Much to our surprise, some studies have proposed that fluoroquinolones increase the incidence of aortic dissection or aortic aneurysm rupture. In 2018, FDA concluded that the evidence on associations between fluoroquinolones use and aortic aneurysm or dissection is consistent in epidemiological studies ${ }^{[5]}$. Other authors $^{[6]}$ supported that ciprofloxacin increases susceptibility to $A A D$ formation and aortic rupture in a mouse model. In these mice, ciprofloxacin suppressed the expression of lysyl oxidase (LOX), increased the level of matrix metalloproteinase (MMP), and increased the rupture of elastic fibers, which may contribute to increase susceptibility to aortic destruction.

\section{Conclusion}

Ciprofloxacin is widely used in general infected population, including infectious aortic dissection patients. Meanwhile, patients with aortic arch surgery are more likely to have postoperative neurological symptoms. Therefore, ciprofloxacin should be used with caution in the risk of drug-induced psychosis and drug-induced residual dilated aortic rupture, especially in patients with aortic dissection before and after surgery.

\section{Declarations}


Consent for publication: Yes

Availability of data and materials: Not applicable

Competing interests: The authors declare that they have no competing interests

Funding: No funding

Authors' contributions: Dr. Song and Jiang participated in the operation and follow-up treatment of the patients. Dr. Tan participated in the guidance of antibiotics and the diagnosis of drug-induced psychosis. Dr. Li participated in the whole process of the patient's treatment, and was the major contributor in writing the manuscript.

Acknowledgements: Not applicable

\section{References}

[1] Grimm O, Alm B, Für Seelische Z. A case of ciprofloxacin-induced acute polymorphic psychosis with a distinct deficit in executive functions. Psychosomatics. 2007;48(3):269

[2] Ben-Chetrit E, Rothstein N, Munter G. Ciprofloxacin-induced psychosis. Antimicrob Agents Chemother. 2013;57(8):4079.

[3] Ranjan A, Praharaj SK. Ciprofloxacin-induced psychosis. J Neuropsychiatry Clin Neurosci. 2014;26(1):e36-7.

[4] Kaur K, Fayad R, Saxena A, et al. Fluoroquinolone-related neuropsychiatric and mitochondrial toxicity: a collaborative investigation by scientists and members of a

social network. J Community Support Oncol. 2016;14(2):54-65.

[5] Bennett AC, Bennett CL, Witherspoon BJ, et al. An evaluation of reports of ciprofloxacin, levofloxacin, and moxifloxacin-association neuropsychiatric toxicities, long-term disability, and aortic aneurysms/dissections disseminated by the Food and Drug Administration and the European Medicines Agency. Expert Opin Drug Saf. 2019;18(11):1055-1063.

[6] LeMaire SA, Zhang L, Luo W, et al. Effect of Ciprofloxacin on Susceptibility to Aortic Dissection and Rupture in Mice. JAMA Surg. 2018;153(9):e181804 